\title{
Left Spigelian Hernia in Super-Obese Patient: A Case-Report
}

\author{
Duilio Pagano ${ }^{*}, 1$, Gabriel J. Echeverri ${ }^{1}$, Carlo Bartoccelli ${ }^{1}$, Calogero Ricotta ${ }^{1}$, Giuseppe Giuffrida ${ }^{1}$, \\ Michela De Martino ${ }^{1}$, Roberta Cavallin ${ }^{1}$ and Salvatore Gruttadauria ${ }^{1,2}$
}

${ }^{I}$ Istituto Mediterraneo Trapianti e Terapie ad Alta Specializzazione, University of Pittsburgh Medical Center in Italy, Palermo, Italy

${ }^{2}$ Department of Surgery University of Pittsburgh, Pittsburgh, PA, USA

\begin{abstract}
We report a case in which a giant Spigelian hernia presented with severe, persistent abdominal pain in a $62-$ year-old super-obese woman with a history of total abdominal hysterectomy-bilateral salpingo-oopherectomy at the age of 40 years for benign neoplasia, who later underwent open hernia repair (maximal diameter of the hernia sac: $21 \mathrm{~cm}$ ) because of the development of compartment syndrome. Afterward, although a large incarcerated hernia disease was found, the sac removal was technically difficult and an open procedure was carried out, with double-mesh placement. No complications were recorded during the post-operative course.
\end{abstract}

We report a difficult issue related to the diagnosis and management of this pathology, underlining the complete surgical repair to avoid hernia recurrence.

Keywords: Spigelian hernia, super-obesity, abdominal wall, hernia repair.

\section{INTRODUCTION}

Spigelian hernia develops in an anterolateral area of the abdomen between the anterior superior iliac spine and the umbilicus in proximity to the external margin of the rectus large muscle, resulting in a relative tenderness of the wall. We report a difficult issue related to the diagnosis and management of this pathology, underlining the complete surgical repair to avoid hernia recurrence.

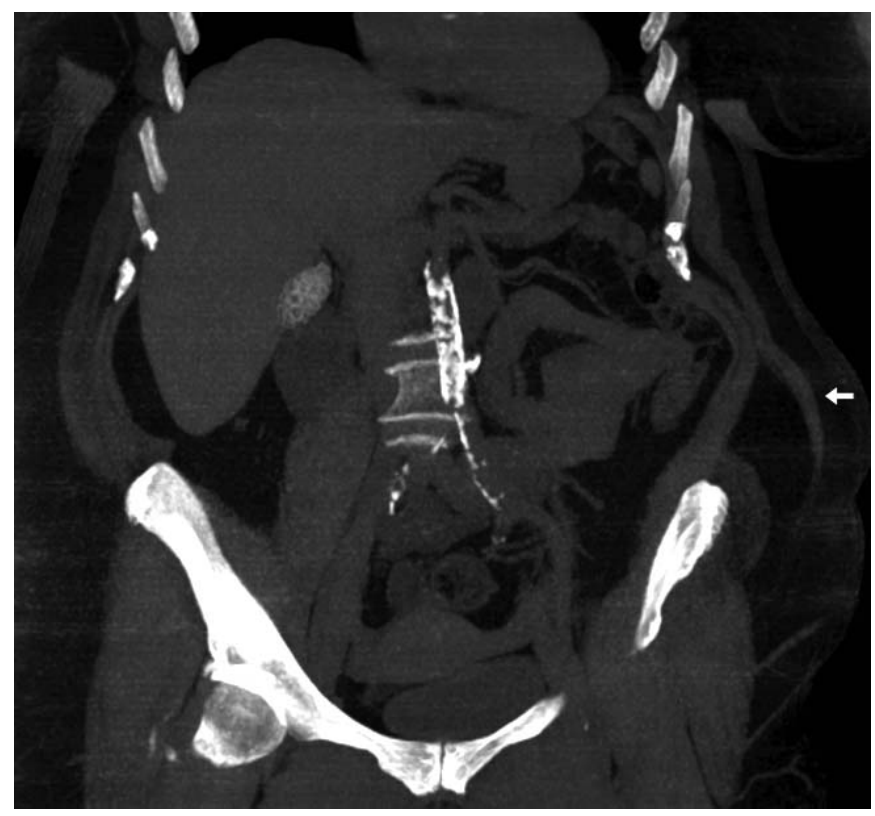

Fig. (1). A coronal image of the abdomen shows incarcerated left spigelian hernia (white arrow).

abdominis muscle, which topographically corresponds to the arcuate line of Douglas; this area is characterized by a specific anatomic arrangement of the sheaths of the abdominal

*Address correspondence to this author at the Istituto Mediterraneo Trapianti e Terapie ad Alta Specializzazione, University of Pittsburgh Medical Center in Italy, Via E. Tricomi, 190127 Palermo, Italy; Tel: +390912192111; Fax: +390912192400; E-mail: dpagano@ismett.edu

\section{CASE REPORT}

A 62-year-old woman was evaluated for a 7-month history of intermittent, crampy, abdominal pain. The patient complained several comorbidities: morbid obesity (BMI 45.2 $\mathrm{kg} / \mathrm{m}^{2}$ ), hypertension, asthma and obstructive sleep apnea, dyslipidemia, cholelithiasis and gastroesophageal reflux disease. She had a history of total abdominal hysterectomy- 


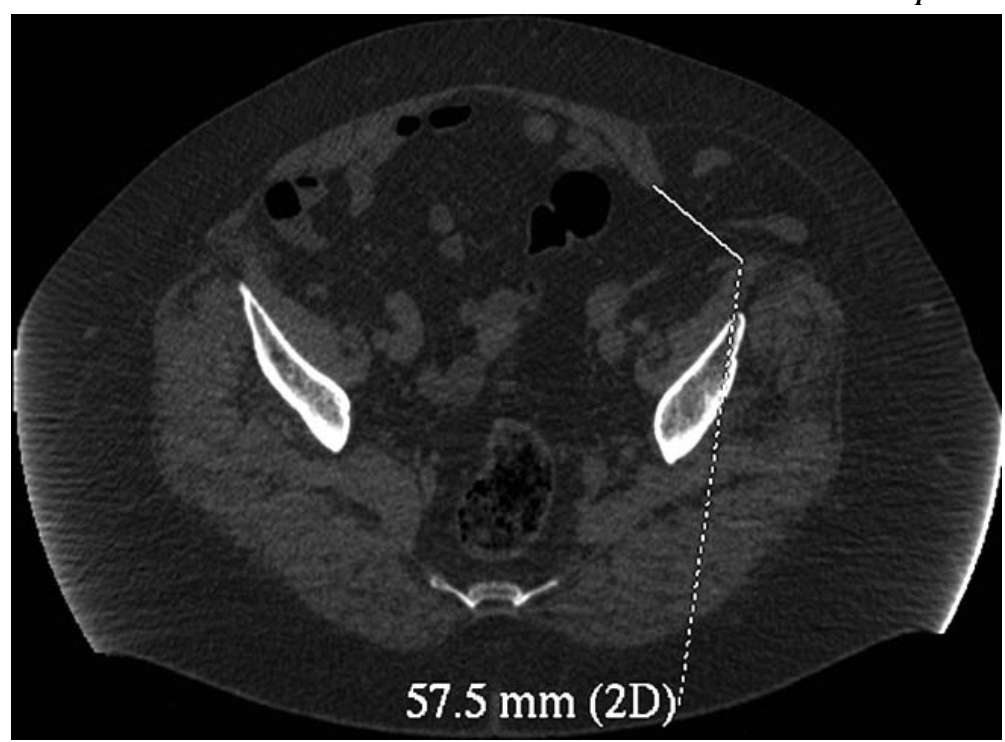

Fig. (2). A sagittal image of the abdomen shows the abdominal wall defect (diameter of $57.5 \mathrm{~mm}$ ).

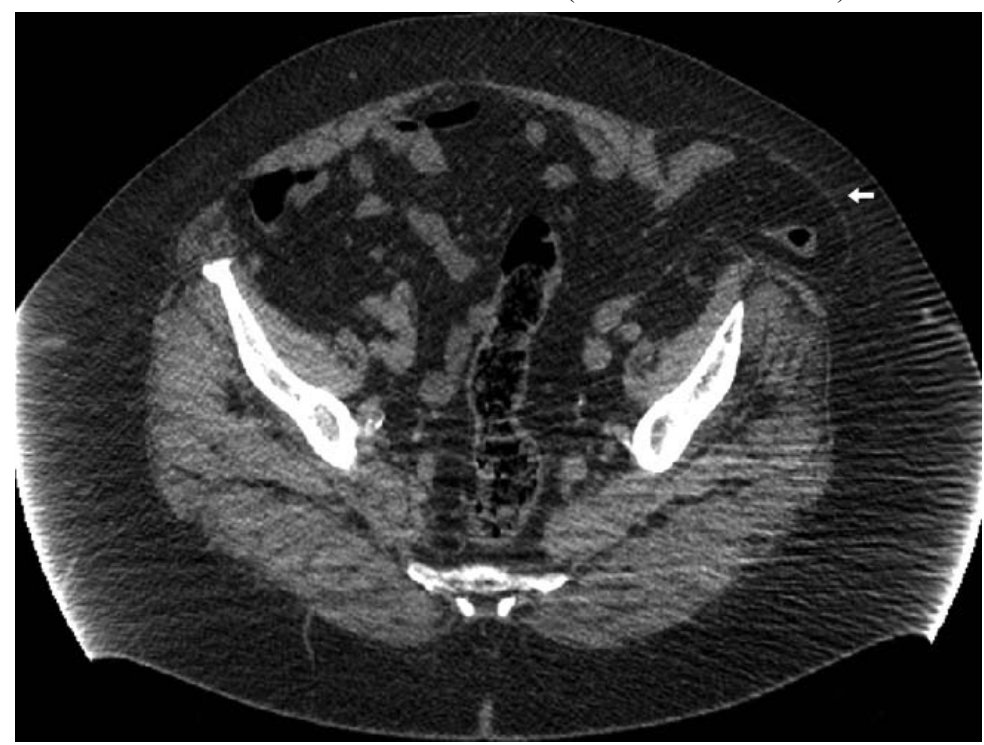

Fig. (3). A sagittal image of the abdomen shows the abdominal hernia sac (white arrow).

bilateral salpingo-oopherectomy at the age of 40 years for benign neoplasia. On physical examination, a large tender bulge was found on the left side of the patient's abdomen that was most prominent while the patient was standing. An abdominal computed tomography scan showed a left-sided incarcerated spigelian hernia (Figs. 1 and 2). Because of these several comorbidities, we decided an open approach and mesh placement through a left lateral mini-laparotomy (8 $\mathrm{cm}$ in length) (Fig. 3).

The procedures were performed without any blood loss and the difficult excision of the hernia sac (maximal diameter of $21 \mathrm{~cm}$ ) induced us to accurately isolate it and, without opening it, to reduce into the abdominal cavity (Fig. 4).

A mesh was placed in the preoperational space, and it was anchored anteriorly to the flat muscles of the abdominal wall and their aponeuroses. The aponeuroses of both the oblique major and of the oblique minor were closed. The suturing respected the consequence of the anatomical layers (the muscular aponeurosis and subcutaneous tissue).
The postoperative course was uncomplicated and an objective evaluation in association with an echo tomography of the abdomen performed three months later did not show any recurrence or any signs of complications.

\section{DISCUSSION}

This is an uncommon ventral hernia characterized by a defect in the linea semilunaris and has also been known as "hernias through the conjoint tendon". It may develop anywhere in the longitudinal area between the lateral border of the rectus abdominis and the medial border of the transverses abdominis muscles. The majority occur within the spigelian hernia belt, the $6-\mathrm{cm}$ area of spigelian aponeurosis that lies cephalad to the interspinal plane [1].

With the advances in radiological imaging, an increase has occurred in the number of cases diagnosed. The predisposing factors resulting in increased intra abdominal pressure and factors that attenuate the tone of the oblique muscles and connective tissue integrity are the same as for other types of hernias [2]. 


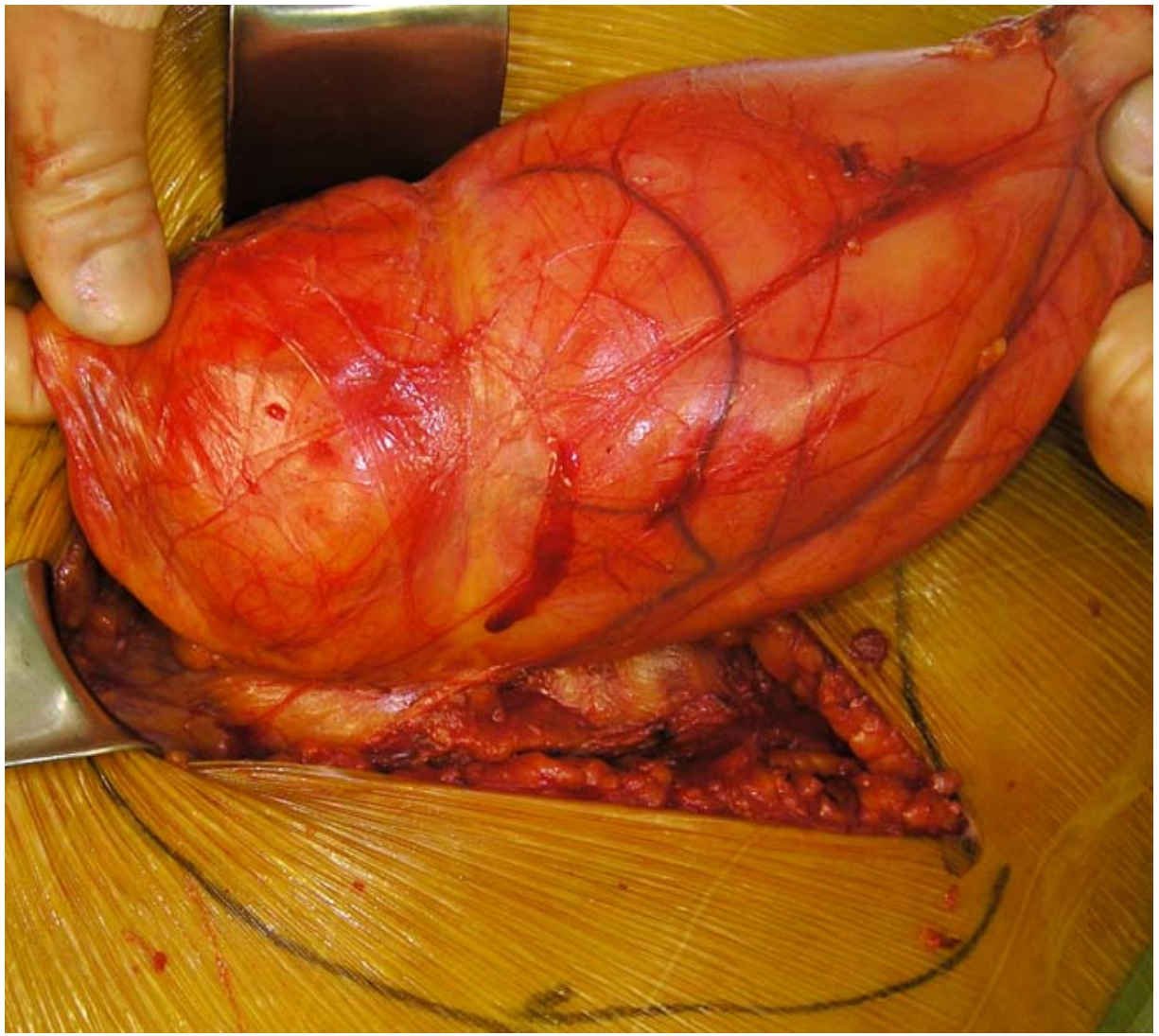

Fig. (4). Hernia sac (maximal diameter of $21 \mathrm{~cm}$ ).

The lateral ventral defect repair was accomplished via an open transverse incision (Fig. 4) and a composite mesh fixation. Postoperatively, the patient's symptoms resolved. Follow-up examination ruled out the hernia recurrence. Apparently, an open Trans abdominal repair with either suture or mesh placement would cause more debility, as patients usually require a longer recovery time after this approach. Usually, patients operated on through laparoscopy or through a preoperational approach recover faster and return to normal activities in as few as 8-10 days [3].

Spigelian hernias should always be operated due to the high risk of incarceration-related complications. The association of laparoscopic ventral hernia repair with decreased tissue trauma and minimized exposure of the prosthetic material to the environment may account for the decreased infection and recurrence rates. The decrease in recurrence rates may be attributable to the technical advantages of the laparoscopic approach. These include wider coverage with the mesh and the ability to identify all defects and weak areas in the fascia. It is possible, however, that shorter follow-ups (averaging 2-3 years) explain the lower recurrence rates reported in the laparoscopic series [4].

In this case the type of repair was chosen according to the patients' comorbidities, but it seems likely that preperitoneal repairs, with open approach, yield shorter hospital stays and better patient compliance.

\section{REFERENCES}

[1] Bennett D. In: Fitzgibbons RJ, Greenburg AG, Eds. Nyhus and Condon's hernia. $5^{\text {th }}$ ed. Philadelphia, Lippincott Williams \& Wilkins 2002; 389-413.

[2] Moreno-Egea A, Flores B, Girela E, Martin JG, Aguayo JL, Canteras M. Spigelian hernia: bibliographical study and presentation of a series of 28 patients. Hernia 2002; 6: 167-70.

[3] Savarise MT, Simpson JP, Moore JM, Leis VM. Improved functional outcome and more rapid return to normal activity following laparoscopic hernia repair. Surg Endosc 2001; 15: 574-8.

[4] Toy FK, Bailey RV, Carey S, et al. Prospective multicenter study of laparoscopic ventral hernioplasty. Preliminary results. Surg Endosc 1998; 12: 955-9. 